\title{
Increased urine interleukin (IL)-17 and IL-22 in patients with Candida urinary tract infection
}

\author{
Mohammad Kord, ${ }^{1}$ Kazem Ahmadikia, ${ }^{1}$ Shahram Mahmoudi, ${ }^{1}$ Mohammad \\ Yarahmadi, ${ }^{1}$ Omid Raiesi ${ }^{1}$
}

\section{Department of Medical Parasitology and Mycology, School of Public Health, Tehran University of Medical Sciences, Tehran, Iran}

Objectives: Candiduria is a common finding in the hospitalized patients. The goal of this study was to quantify interleukin (IL)-17 and IL-22 levels in urine of candiduric and non-candiduric subjects.

Methods: A case control study was conducted. Urine specimens were obtained from 338 patients hospitalized in Hashemi Nejad Kidney Center over nine months. Thirty four patients who fulfilled the inclusion criteria (>103 CFU/ml and presence of only Candida species in urine specimen) were enrolled as case group and urine samples with concomitant infections were excluded. Thirty four patients with negative direct examination and culture were included as control patients. Then IL-17 and IL-22 were measured in the lyophilized and non lyophilized urine. The relevant cytokine titers of the two groups were compared and the associated of cytokines elevation and candiduria was investigated.

Results: The majority of candiduric patients were from ICU and urology units of women. Only 4 patients (11.7\%) manifested fever and dysuria. Massive leukocyturia (>50 WBC per hpf) was observed in urine of 4 patients(Table 1). Candida glabrata was commonly isolated species (44\%)(Table 2). Levels of the urine IL17 and IL-22 were significantly elevated in candiduric patients when compared to non-candiduric control subjects. While an increased IL-17 level was significantly associated with candiduria \{Odds ratio1.09, $95 \%$ confidence interval 1.003 - 1.17; $\mathrm{P}=0.04\}$, an increased IL-22 level was not. The results showed that lyophilized urine samples will maximize the detection power of urinary cytokines (Table 3).
Table 1. Characteristics of Patients With Candiduria and Control Group

\begin{tabular}{lccc}
\hline \multicolumn{1}{c}{ Variables } & $\begin{array}{c}\text { Case } \\
\text { group(n=34) } \\
\mathbf{N}(\%)\end{array}$ & $\begin{array}{c}\text { Control } \\
\text { group }(\mathbf{n}=\mathbf{3 4}) \\
\mathbf{N}(\%)\end{array}$ & p value \\
\hline Mean age in years(range) & $59.5(17-89)$ & $50.7(21-65)$ & $\mathbf{0 . 0 3 7}$ \\
Sex(Male/Female) & $14 / 20$ & $16 / 18$ & NS \\
Foley catheter & $21(61.8)$ & $16(47.1)$ & NS \\
Diabetes & $19(55.9)$ & $2(5.9)$ & $\mathbf{0 . 0 0 0 1}$ \\
Prior antibiotics usage & $12(35.2)$ & $1(2.9)$ & $\mathbf{0 . 0 0 1}$ \\
Hospitalized within at least 6 & $4(11.7)$ & 0 & NS \\
months & & & \\
Mortality & $7(20.5)$ & 0 & $\mathbf{0 . 0 1 1}$ \\
Central venous line & $8(23.5)$ & 0 & $\mathbf{0 . 0 0 5}$ \\
Hospitalized in ICU & $12(35.2)$ & 0 & $\mathbf{0 . 0 0 1}$ \\
Dialysis & $1(2.9)$ & 0 & $\mathbf{N S}$ \\
Renal transplantation & $2(5.9)$ & 0 & $\mathbf{N S}$ \\
Heavy leukocyturia(>50 WBC & $4(11.7)$ & 0 & $\mathbf{N S}$ \\
per hpf) & & & \\
Fever and dysuria & $4(11.7)$ & 0 & $\mathbf{N S}$ \\
Mental confusion & $5(14.7)$ & 0 & $\mathbf{0 . 0 5 3}$ \\
Surgery & $8(23.5)$ & $10(29.4)$ & $\mathbf{N . S}$ \\
\hline
\end{tabular}

Table 2. Candida Species in Subgroups of Patients with Candiduria

\begin{tabular}{|c|c|c|c|c|c|}
\hline \multirow[t]{2}{*}{ Patients subgroups } & \multicolumn{4}{|c|}{ Candida species } & \multirow[b]{2}{*}{$\begin{array}{c}\text { More } \\
\text { than } \\
\text { one } \\
\text { Candida } \\
\text { species } \\
\mathrm{N}(\%)\end{array}$} \\
\hline & & $\begin{array}{c}\text { C.glabrata } \\
\mathrm{N}(\%)\end{array}$ & $\begin{array}{c}C . \\
\text { tropicalis } \\
\mathrm{N}(\%)\end{array}$ & $\begin{array}{c}\text { C.krusei } \\
\mathrm{N}(\%)\end{array}$ & \\
\hline patients in case group $(n=34)$ & $9(26.4)$ & $15(44)$ & 3(9) & $1(3)$ & $6(17.6)$ \\
\hline Diabetic patients $(\mathrm{n}=19)$ & $4(22)$ & $9(47)$ & $2(10)$ & 0 & $4(21)$ \\
\hline Catheterized patients $(n=21)$ & $4(19)$ & $10(47.5)$ & $2(10)$ & 0 & $6(23.5)$ \\
\hline $\begin{array}{l}\text { Patients with prior antibiotic } \\
\text { usage }(n=12)\end{array}$ & $3(25)$ & $3(25)$ & $2(16.3)$ & 0 & $4(33.7)$ \\
\hline Female gender $(n=20)$ & $4(20)$ & $9(45)$ & $3(15)$ & $1(5)$ & $3(15)$ \\
\hline
\end{tabular}

Table 3. Associations Between Increased Lyophilized Urine Levels of Interleukins and Candiduria

\begin{tabular}{l|cc}
\hline \multicolumn{1}{c}{ Cytokine(pg/ml) } & Multiple analysis & P values \\
\cline { 2 - 3 } & Adjusted OR(95\% Cl) & \\
\hline $\mathrm{IL}-17$ & $1.09(1.003-1.17)$ & $\mathbf{0 . 0 4}$ \\
$\mathrm{IL}-22$ & $1.03(0.987-1.07)$ & $\mathbf{0 . 1 9}$ \\
\hline
\end{tabular}

Odds ratios (OR) were adjusted for age, sex and indwelling Foley.

$\mathrm{Cl}=$ confidence interval

Conclusions: Our results indicated that direct examination, fungal urine culture, and investigation of urine IL17 and IL-22 levels are useful tools for diagnosis of Candida urinary tract infection.

Keywords: urinary tract infections, candida, interlukin 17, candiduria 